\title{
Protein Function Prediction using Multi-label Ensemble Classification
}

\author{
Guoxian Yu, Huzefa Rangwala, Carlotta Domeniconi, Guoji Zhang, and Zhiwen Yu, Member, IEEE
}

\begin{abstract}
High-throughput experimental techniques produce several kinds of heterogeneous proteomic and genomic datasets. To computationally annotate proteins, it is necessary and promising to integrate these heterogeneous data sources. Some methods transform these data sources into different kernels or feature representations. Next, these kernels are linearly (or non-linearly) combined into a composite kernel. The composite kernel is utilized to develop a predictive model to infer the function of proteins. A protein can have multiple roles and functions (or labels). Therefore, multi-label learning methods are also adapted for protein function prediction.

We develop a transductive multi-label classifier (TMC) to predict multiple functions of proteins using several unlabeled proteins. We also propose a method called transductive multi-label ensemble classifier (TMEC) for integrating the different data sources using an ensemble approach. TMEC trains a graph-based multi-label classifier on each single data source and then combines the predictions of the individual classifiers. We use a directed bi-relational graph to capture the relationships between pairs of proteins, between pairs of functions, and between proteins and functions. We evaluate the effectiveness of TMC and TMEC to predict the functions of proteins on three benchmarks. We show that our approaches perform better than recently proposed protein function prediction methods on composite and multiple kernels. The code, datasets used in this paper and supplementary file are available at https://sites.google.com/site/guoxian85/tmec
\end{abstract}

Index Terms-Multi-label Ensemble Classifiers, Directed Bi-relational Graph, Protein Function Prediction

\section{INTRODUCTION}

A DVANCES in biotechnology have enabled highthroughput experiments that generate high volume of genomic and proteomic data. Examples of these data include protein-protein interaction (PPI) networks, protein sequences, protein structure, gene co-expression data, and genetic interaction networks. Each data source provides a complementary view

G. Yu is with the College of Computer and Information Science, Southwest University, Chongqing, 410075 China, and School of Computer Science and Engineering, South China University of Technology, Guangzhou, 510006 China, email: gxyu@swu.edu.cn

H. Rangwala and C. Domeniconi are with the Department of Computer Science, George Mason University, Fairfax, VA, 22030 USA, email: rangwala@cs.gmu.edu, carlotta@cs.gmu.edu

G. Zhang is with the School of Sciences, South China University of Technology, Guangzhou, 510640 China, email: magjzh@scut.edu.cn

$\mathrm{Z}$. Yu is with the School of Computer Science and Engineering, South China University of Technology, Guangzhou, 510006 China, email: zhwyu@scut.edu.cn

Manuscript received 4 Apr. 2013; revised 17 Aug. 2013; accepted 29 Aug. 2013; published online $x x x x x .2013$ of the underlying mechanisms within a living cell. Annotating the functions (i.e., biological process functions in the Gene Ontology (GO) [1], transcription and protein synthesis) of proteins is a fundamental task in the post-genomic era [2], [3]. However, it is time consuming, expensive, and low productive to manually annotate a protein using complex and large heterogeneous data. Therefore, various computational models have been proposed to automatically infer the functions of proteins by integrating the available data [3], [4].

Kernel based methods [5], [6] have been widely used to design several bioinformatics related algorithms. In these approaches, the pairwise similarity between proteins is described by a kernel function $\mathcal{K}$, which captures the underlying biological complexity associated with the proteins. For each data source (i.e., protein sequences, PPI networks), a unique kernel function is defined, and each kernel function captures a different notion of similarity. For example, the string kernel [7] is often used to compute the similarity between protein sequences, and the random walk kernel [2] is utilized on protein-protein interaction (PPI) data. Both the sequence and PPI datasets are transformed into different kernels, each of which captures similarities between protein pairs within different feature spaces (or embeddings). Pavlidis et al. |8| and Noble et al. [4] observed that the prediction accuracy can be boosted by taking advantage of complimentary embeddings across different kernels. Many approaches [5], [9], [10] use a linear (or nonlinear) weighted combination (optimal or ad hoc) of multiple kernels obtained from the different sources. These kinds of methods can be categorized as kernel integration methods. In addition, supervised ensemble classifiers [8], [11] have also been developed to combine the multiple data sources.

Often, only a few proteins are annotated, and a large volume of proteins remains unlabeled within each single data source. Transductive or semi-supervised learning methods are able to make use of unlabeled data to boost the learning results [12]. Therefore, several semi-supervised [10], [13], [14] approaches have been proposed for protein function prediction. Further, a protein often holds more than one function and functions are correlated with each other. This 
fact should be taken into account in protein function prediction. Several approaches $\mid[15|-| 18 \mid$ formulate the protein function prediction problem within a multilabel learning framework. Multi-label learning methods can make use of the dependencies between the different function classes, and they often outperform single-labeled prediction methods.

We propose a protein function prediction method called Transductive Multi-label Classifier (TMC). TMC is based on a directed bi-relational graph that models the relationship between proteins and functions. To integrate the heterogeneous sources of protein data, we also develop an ensemble based classifier called Transductive Multi-label Ensemble Classifier (TMEC). We performed comprehensive experiments evaluating the performance of TMC and TMEC on three protein function prediction benchmarks (Yeast, Human and Fly). Each benchmark includes several kinds of heterogeneous data sources (i.e., PPI networks, protein sequences and gene co-expression data). For more information on these benchmarks, one can refer to prior work by Mostafavi et. al. [9] 17. Our results show that the use of a directed bi-relational graph achieves higher accuracy than the undirected one. Our proposed TMC outperforms state-of-the-art protein function prediction approaches; namely, two transductive multi-label classification approaches [15], [18], a transductive classifier |9| and two multi-label multiple kernel learning methods [19], [20]. In addition, the proposed TMEC, which takes advantage of classifier ensembles to make use of multiple heterogeneous data sources, often performs better than TMC.

This work is an extension of our earlier paper by $\mathrm{Yu}$ et al. [21]. In particular, the additional contributions of this paper are as follows.

1) We provide a thorough analysis of the issues due to the use of an undirected bi-relational graph [22] for network propagation. We advocate the use of a directed bi-relational graph instead, and discuss a toy example to illustrate the risk of using an undirected bi-relational graph.

2) We test our proposed methods (TMC and TMEC) on three public available protein function prediction benchmarks (Yeast, Human, and Fly).

3) We apply different schemes to combine the individual kernels from different data sources into a composite kernel, and compare them against TMEC to further show the advantage of classifier integration to kernel integration in protein function prediction.

The rest of the paper is organized as follows. Section 2 reviews related work on protein function prediction using multi-label learning and data integration. In Section 3. we introduce the directed bi-relational graph approach and the corresponding training procedure, along with a toy example to illustrate the drawbacks

1. http://morrislab.med.utoronto.ca/ sara/SW/ associated with the use of an undirected bi-relational graph. We also describe our ensemble approach to make use of multiple data sources. We describe the experimental protocol, evaluation metrics, and data sources in Section 4 In Section 5 , we discuss the experimental results. In Section 6, we provide conclusions along with directions for future work.

\section{Related Work}

Various computational models have been proposed to predict protein functions. These methods can be categorized according to terms of methodology, input data, and problem definition. Pandey et al. 3] gave a comprehensive literature review on protein function prediction. Here, we review only the work most related to the scope of this paper.

\subsection{Multi-label Learning in Protein Function Pre- diction}

Traditional function prediction methods take protein sequences (or PPI) and functional annotations as input to train one-versus-the-rest binary classification models [2], [8]. These methods ignore the pairwise function correlations. It is observed that a protein performs multiple functions, and structured relationships are prevalent within protein function annotation databases (e.g., Gene Ontology $|1|^{2}$ is a directed acyclic graph, Functional Catalogue (FunCat) [23] $]^{3}$ is a tree graph). As such, protein function prediction can be formulated as a multi-label classification problem [24].

Multi-label learning is widely used in protein function prediction. Elisseeff et al. [25] added a ranking loss function into the loss function of SVMs and proposed a method called RankSVM. RankSVM does not to make explicit use of the label correlations [26], which can be important for deciding the label membership [18], [26]. Chen et al. [26] incorporated a label correlation (captured by a hypergraph) term into the objective function of RankSVM. More specifically, in the hypergraph, each label corresponds to a hyperedge, which connects all the proteins that share the same label. Protein function databases like Gene Ontology [1] and FunCat [23] represent protein functions within a hierarchy (or a directed acyclic graph), and several approaches incorporate the parentchildren relationship among proteins. Barutcuoglu et al. [27], first train independent binary SVMs for each of the GO function labels, and then integrates the prediction by incorporating GO's hierarchical structure within a Bayes formulation. Valentini [28] used the True Path Rule in FunCat to guide the integration of predictions. Pandey et al. |17| used Lin's measure [29] to define the semantic similarity between the different

2. http://www.geneontology.org/

3. http://mips.helmholtz-muenchen.de/proj/funcatDB/ 
GO labels and incorporated it within a weighted multilabel $k$-Nearest Neighbors $(k \mathrm{NN})$ classifier.

More recently, several semi-supervised multi-label learning methods based on PPI networks were proposed for protein function prediction [15], [16], [18]. MCSL [16] is based on a product graph with an objective function similar to the local and global consistency method [30]. MCSL adds another term (function correlation) into the smoothness term (See Eq. (6) in [16|) and results in a Kronecker matrix. For $N$ proteins with $C$ functions, the resulting Kronecker matrix is an $(N \times C) \times(N \times C)$ matrix. This augmented matrix often can not be loaded in memory and it's computationally expensive. To address this problem, Jiang [15] proposed a protein function prediction approach called PfunBG, which is based on a birelational graph [22] and label propagation [30]. The birelational graph captures three types of relationships: (i) protein-protein similarities, (ii) function-function similarities, and (iii) protein-function associations, and the association matrix of the bi-relational graph is an $(N+C) \times(N+C)$ matrix. GRF [18] utilizes Jaccard coefficients to measure the correlation between functions and then incorporates this correlation into a general semi-supervised learning framework based on manifold regularization [31]. All the described methods, MCSL, PfunBG and GRF, utilize pairwise label correlations within a semi-supervised learning framework, but are developed for protein function prediction using a single data source (PPI) only.

\subsection{Data Integration in Protein Function Predic- tion}

Several methods have been developed to integrate the information from heterogeneous data sources (i.e., PPI, protein sequences) to boost the function prediction accuracy [4]. Lanckriet et al. [5] defined a kernel on each data source and then utilized semi-definite programming (SDP) to seek the optimal weights to linearly combine these kernels. However, this method is computationally expensive [10]. To overcome this problem, Tsuda et al. [10] made use of the dual problem and gradient descent to efficiently get the optimal weights. Shin et al. [13] sought the optimal weights within an EM [32] framework by iteratively minimizing prediction error and combining weights. Mostfavi et al. [33] propose a heuristic approach derived from ridge regression, to more efficiently determine these weights. Finally, these obtained composite kernels are used in SVMs or graph-based classifiers for binary protein function annotation. These methods determine the set of weights per function class, which not only result in increased time complexity, but also ignore the inherent correlation among function labels.

More recently, some approaches have leveraged kernel integration and function correlation. Mostafavi et al. [9] introduced a method called 'Simultaneous
Weighting (SW)'. SW optimizes a set of weights for a group of correlated functions, and then combines these kernels into a composite kernel. Next, graphbased semi-supervised classifiers are trained on this composite kernel for each function. Tang et al. [19] introduced a unified framework, in which selecting a specific composite kernel for each function and for all the functions are two extreme cases. In our experiments, we consider this approach for comparison, and call it MKL-Sum [19]. MKL-Sum first learns linear combination coefficients with respect to all the functions and then applies SVMs on the composite kernel. Bucak et al. [20] utilized stochastic approximation to speedup multi-label multiple kernel learning, and proposed a method called MKL-SA. Cesa-Bianchi et al. [34] integrated binary classifiers hierarchical ensembles, cost sensitive methods, and data fusion for gene function prediction. However, despite various optimization techniques, Tsuda et al. [10], Lewis [6] and Gönen et al. [35] observed that a composite kernel combined with optimized weights has similar performance to a composite kernel combined with equal weights, i.e., without optimization.

In this work, different from traditional kernel integration methods [5], [9], [19], we propose a classifier integration method called TMEC. TMEC first trains a transductive multi-label classifier (TMC) on each of the kernels representing a data source, and then integrates the predictions using majority voting. Our experimental results on three public available protein function prediction benchmarks show that TMEC outperforms classifiers trained on the composite kernels. In addition, we observe that TMEC outperforms the TMC trained on the composite kernel and the individual kernels derived from different data sources. This observation confirms that the ensemble approach is effective, and the transductive multi-label classifiers trained on individual kernels are complementary to each other.

\section{Problem Formulation}

We are given $R$ different kinds of features that describe the same set of $N$ proteins with $C$ functions. Each kind of features provide a unique representation for proteins (e.g. vectors, trees, or networks). We assume the first $l$ proteins are already annotated and the remaining $u$ proteins are not annotated $(l+u=N)$. The $R$ different representations of these proteins are transformed into $R$ kernels $\left[K_{r}\right]_{r=1}^{R}\left(K_{r} \in \mathbb{R}^{N \times N}\right)$, one kernel per source. $K_{r}(i, j) \geq 0$ describes the kernel induced pairwise similarity between proteins $i$ and $j$ in the $r$-th data source. Our objective is to first train a TMC on a directed bi-relational graph adapted from the kernel $K_{r}$, and then combine these classifiers into an ensemble classifier (TMEC). Finally, we use TMEC to annotate these $u$ proteins. In this section, we first review the birelational graph approach, analyze the related issues 
for network propagation, and give a toy example to illustrate this problem.

\subsection{Transductive Multi-label Classification on a Directed Bi-relational Graph}

Graph based transductive or semi-supervised learning methods can be extended to multi-label learning by incorporating a label correlation term into its objective function [16], [18], [36]. Wang et al. [22] introduced an undirected bi-relational graph for image classification and applied a random walk based propagation with restart [37]. This graph includes both images and labels as nodes. For consistency, hereinafter, we use proteins instead of images and functions instead of labels. A birelational graph is composed of three kinds of edges: between proteins, between functions, and between proteins and functions. For the latter, if protein $i$ has function $c$, an edge is set between them.

The inter-function similarity leads to improved prediction accuracy [17], [18] and can be defined in various ways [15], |17], [18]. Here we define the similarity between functions $m$ and $n$ as follows:

$$
S_{F F}(m, n)=\frac{\mathbf{f}_{m}^{\mathrm{T}} \mathbf{f}_{n}}{\left\|\mathbf{f}_{m}\right\|\left\|\mathbf{f}_{n}\right\|}
$$

where $\mathbf{f}_{m} \in \mathbb{R}^{N}(1 \leq m \leq C)$ is the $m$-th function vector on all proteins: if protein $i$ has function $m$, then $\mathbf{f}_{m}(i)=1$, otherwise $\mathbf{f}_{m}(i)=0$.

A random walk on a graph is often described by a propagation matrix. For a random walk on a birelational graph, the propagation matrix $W$ is defined as:

$$
W=\left[\begin{array}{cc}
\beta W_{P P} & (1-\beta) W_{P F} \\
(1-\beta) W_{F P} & \beta W_{F F}
\end{array}\right]
$$

where $W_{P P} \in \mathbb{R}^{N \times N}$ and $W_{F F} \in \mathbb{R}^{C \times C}$ are the propagation matrices of the intra-subgraphs of proteins and functions, respectively. $W_{P F} \in \mathbb{R}^{N \times C}$ and $W_{F P} \in \mathbb{R}^{C \times N}$ are the inter-subgraph propagation matrices between proteins and functions, and $\beta$ controls the relative importance of the intra- and the intersubgraphs. It also controls the frequency with which a random walker jumps from a function subgraph to a protein subgraph. $W_{P P}$ and $W_{F F}$ are computed as:

$$
W_{P P}=D_{P P}^{-1} S_{P P} \quad W_{F F}=D_{F F}^{-1} S_{F F}
$$

where $S_{P P}$ is the pairwise similarity matrix between all proteins, and $S_{F F}$ is the pairwise correlation matrix between all functions. $D_{P P}$ and $D_{F F}$ are the diagonal matrices of the row sums of $S_{P P}$ and $S_{F F}$, respectively. $W_{P F}$ and $W_{F P}$ are calculated as:

$$
W_{P F}=D_{P F}^{-\frac{1}{2}} S_{P F} D_{F P}^{-\frac{1}{2}} \quad W_{F P}=D_{F P}^{-\frac{1}{2}} S_{F P} D_{P F}^{-\frac{1}{2}},
$$

where $S_{P F}$ is the relation matrix between proteins and functions, and $S_{F P}$ is the transpose of $S_{P F}$. $D_{P F}$ is the diagonal matrix of the row sums of $S_{P F}$ and $D_{F P}$ is the diagonal matrix of the column sums of
$S_{P F}$. We observe that if protein $i$ has function $c$ then $S_{P F}(i, c)=1$; otherwise $S_{P F}(i, c)=0$.

The $c$-th function node and the proteins annotated with this function are considered as a group:

$$
G_{c}=v_{c}^{F} \cup\left\{v_{i}^{P} \mid S_{P F}(i, c)=1\right\}
$$

where $v_{c}^{F}$ is the $c$-th function node and $v_{i}^{P}$ is the $i$ th protein node of the bi-relational graph. In the birelational graph, instead of computing the node-tonode relevance between a function node and an unannotated protein node, the relevance between a protein and a group $G_{c}$ is considered. Let $\tilde{Y} \in \mathbb{R}^{(N+C) \times C}$ be the label distribution on the $N+C$ nodes of the birelational graph with respect to $C$ function labels. Each column corresponds to one function label. For the $c$-th function, the distribution vector $\tilde{Y}_{. c}(c$-th column of $\tilde{Y})$ is:

$$
\tilde{Y}_{. c}=\left[\begin{array}{c}
\gamma \tilde{Y}_{. .}^{P} \\
(1-\gamma) \tilde{Y}_{. c}^{F}
\end{array}\right] \in \mathbb{R}^{N+C}
$$

where $\tilde{Y}_{. c}^{P} \in \mathbb{R}^{N}$ is the distribution vector on the protein nodes, and $\tilde{Y}_{. c}^{F} \in \mathbb{R}^{C}$ is the distribution vector on the function nodes. $\tilde{Y}_{. c}^{P}(i)=1 / \sum_{i=1}^{N} S_{P F}(i, c)$ if $S_{P F}(i, c)=1$ and $\tilde{Y}_{. c}^{P}(i)=0$ otherwise; $\tilde{Y}_{. c}^{F}(j)=1$ if $j=c$, and $\tilde{Y}_{. c}^{F}(j)=0$ otherwise. $\gamma$ adjusts the distribution of function labels on protein and function nodes.

Based on these preliminaries, an iterative objective function is defined on this bi-relational graph as follows:

$$
F^{(t+1)}(j)=(1-\alpha) \sum_{i=1}^{N+C} W(i, j) F^{(t)}(i)+\alpha \tilde{Y}_{j}
$$

where $F^{(t)}(i) \in \mathbb{R}^{C}$ is the predicted likelihood of the $i$-th protein with respect to $C$ function labels in the $t$-th iteration, $W(i, j)$ is the weight of edge between nodes $i$ and $j, \tilde{Y}_{j} \in \mathbb{R}^{C}$ is the initial set of functions on the $j$-th node, $\alpha$ is a scalar value to balance the tradeoff between the initial set functions and the predicted functions. From Eq. (7), we can see that the functions of a node are predicted by the functions of its connected nodes. This makes TMC a direct protein function prediction method [2]. Note that, in the birelational graph, the predictions are made on all the nodes (including proteins and functions).

However, the application of Eq. (7) for protein function prediction has a major drawback. Suppose $i$ is a protein vertex annotated with a function vertex $j$. The function $j$ may be overwritten by the functions of the proteins connected to $i$, thus causing the loss of reliable information. As such, the functions of initially annotated proteins may be changed during the iterative label propagation. This phenomenon is similar to the one occurring in the local and global consistency method [30], and should be avoided. A toy example in the following section will illustrate this problem. 
Eq. (7) can be rewritten as follows (for simplicity, the parameter $\beta$ in Eq. (2) is not included):

$$
\begin{aligned}
{\left[\begin{array}{l}
F_{P}^{(t+1)} \\
F_{F}^{(t+1)}
\end{array}\right] } & =(1-\alpha)\left[\begin{array}{ll}
W_{P P} & W_{P F} \\
W_{F P} & W_{F F}
\end{array}\right]\left[\begin{array}{l}
F_{P}^{(t)} \\
F_{F}^{(t)}
\end{array}\right]+\alpha\left[\begin{array}{c}
\tilde{Y}_{P} \\
\tilde{Y}_{F}
\end{array}\right] \\
& =(1-\alpha)\left[\begin{array}{l}
W_{P P} F_{P}^{(t)}+W_{P F} F_{F}^{(t)} \\
W_{F P} F_{P}^{(t)}+W_{F F} F_{F}^{(t)}
\end{array}\right]+\alpha\left[\begin{array}{c}
\tilde{Y}_{P} \\
\tilde{Y}_{F}
\end{array}\right] \\
F_{P}^{(t+1)} & =(1-\alpha)\left(W_{P P} F_{P}^{(t)}+W_{P F} F_{F}^{(t)}\right)+\alpha \tilde{Y}_{P} \\
F_{F}^{(t+1)} & =(1-\alpha)\left(W_{F P} F_{P}^{(t)}+W_{F F} F_{F}^{(t)}\right)+\alpha \tilde{Y}_{F}
\end{aligned}
$$

From Eqs. (8) tion information from function to protein nodes, and $W_{F P}$ propagates function information from protein to function nodes. In a bi-relational graph, we expect that information propagates from function to protein nodes, but not vice versa. Thus, we change the undirected bi-relational graph into a directed one. An example of the proposed directed bi-relational graph is shown in Figure 1. In this graph, information can be propagated in the intra-subgraphs $W_{P P}$ and $W_{F F}$, and in the intersubgraph $W_{P F}$, but not in the inter-subgraph $W_{F P}$. Therefore, we define the propagation matrix $W_{d}$ on the directed bi-relational graph as follows:

$$
W_{d}=\left[\begin{array}{cc}
W_{P P} & W_{P F} \\
\mathbf{0} & W_{F F}
\end{array}\right]
$$

where $\mathbf{0} \in \mathbb{R}^{C \times N}$. TMC takes advantage of network propagation by optimizing local and global consistency functions [30]. By defining directed edges between proteins and functions, the induced propagation matrix of the directed bi-relational graph becomes not symmetric. The directed edges control the information that flows from one side of the bipartite graph (function subgraph) to the other (protein sub-graph). As described earlier in Eqs. (8- 9), and through the empirical study in the following Subsection 3.2. we show that TMC trained on a directed bi-relational graph can avoid the observed problems (i.e., annotation change and function label override) associated with TMC trained on an undirected bi-relational graph. Thus, we advocate the use of a directed bi-relational graph, and define a non-symmetric propagation matrix on this graph.

Based on Eq. (7), we can get the iterative equation on the directed bi-relational graph:

$$
F^{(t+1)}=(1-\alpha) W_{d} F^{(t)}+\alpha \tilde{Y}
$$

By setting $F^{(0)}=\tilde{Y}$, we have:

$$
F^{(t+1)}=\left((1-\alpha) W_{d}\right)^{(t+1)} \tilde{Y}+\alpha \sum_{k=0}^{t}\left((1-\alpha) W_{d}\right)^{k} \tilde{Y}
$$

Since $0<\alpha<1$ and $\mathbf{0} \leq(1-\alpha) W_{d}<\mathbf{1}$. Note that when $k=0,\left((1-\alpha) W_{d}\right)^{k}$ is the identity matrix. The first term in Eq. (12) is bound to $\mathbf{0}$, and the second term (excluding $\tilde{Y}$ ) is a geometric series with the following limit:

$$
\lim _{t \rightarrow \infty} \sum_{k=0}^{t}\left((1-\alpha) W_{d}\right)^{k}=\left(I-(1-\alpha) W_{d}\right)^{-1}
$$

where $I$ is an $(N+C) \times(N+C)$ identity matrix. Thus the equilibrium solution $F$ of Eq. (11) is:

$$
F=\alpha\left(I-(1-\alpha) W_{d}\right)^{-1} \tilde{Y}
$$

The predicted $F(j)$ is a real value vector with size $C$, where each entry reflects the likelihood that protein $j$ has the corresponding function. Thus, we also refer to $F(j)$ as the predicted likelihood score vector of protein $j$. From Eq. 14, we can see that $F$ is determined by $W_{d}$ and a well-structured bi-relational graph can produce a competent $F$.

$F_{P}^{(t)}$ is initially set according to the original function annotation on the $l$ annotated proteins. For both the directed and undirected bi-relational graphs, $F_{P}^{(t)}$ is updated in iterations, but $F_{P}^{(t)}$ on the directed bi-relational graph avoids the problems (i.e., annotation change and function label override, c.f. Subsection 3.2) associated with the undirected bi-relational graph, and achieves better prediction. In the undirected bi-relational graph, $W_{F P}$ propagates label information from protein nodes to function nodes, but in the directed bi-relational graph, no information is propagated from the protein nodes to function nodes. Thus, the term $W_{F P} F_{P}^{(t)}$ may lead to poor performance due to $W_{F P}$, while the term $F_{P}^{(t)}$ alone does not have this problem.

\subsection{The Problem of Undirected Bi-relational Graph in Label Propagation}

We provide an illustrative example to instantiate the problem of the undirected bi-relational graph in label propagation, and therefore motivate the use of a directed bi-relational graph.

The toy example is illustrated in Figure 1. In this directed bi-relational graph, there are three function nodes $(f 1, f 2$ and $f 3)$, and six protein nodes $(p 1, \cdots, p 6)$. The first four proteins are labeled (as specified by the directed solid lines), and the last two proteins are unlabeled. In the graph, $p 5$ is more similar to (or interacted with) $p 4$ than $p 3$. From the 'guilty by association' rule [38| (interacting proteins tend to share similar functions), $p 5$ is more likely to have the function set of $p 4$. However, TMC on the undirected birelational graph predicts $p 5$ to have the same function set as $p 3$, instead of $p 4$, as illustrated in the fifth row of Table 1 In contrast, TMC on the directed bi-relational graph predicts $p 5$ to have the same function set as $p 4$.

Another observation is that for the undirected graph, $p 4$ is initially annotated with $f 1$ and $f 3$, but after label propagation, this protein is annotated with $f 1$ and $f 2$ (see the fourth row of Table 1). In addition, after the label propagation on the undirected birelational graph, the information on the $f 3$ node is 


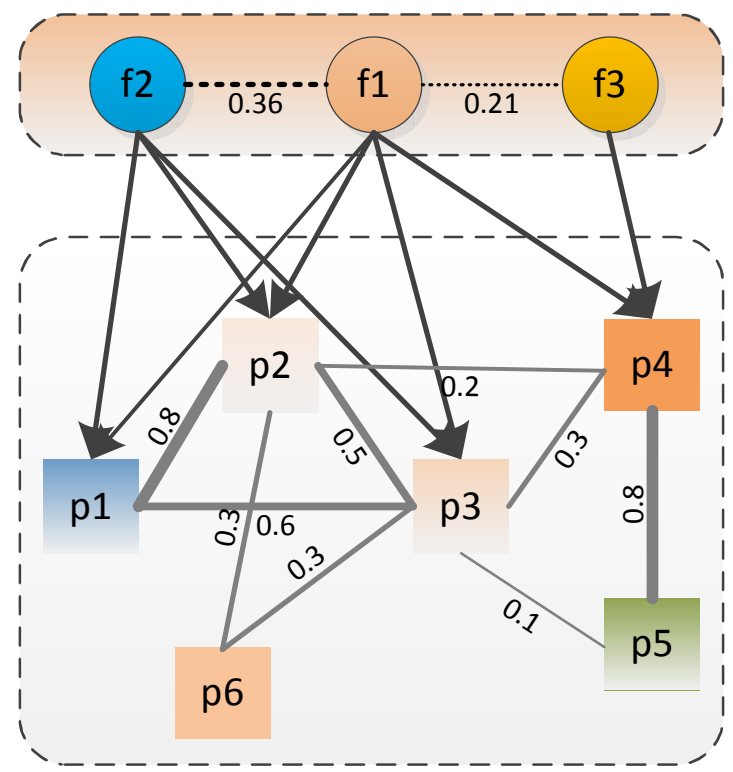

Fig. 1. An example of a directed bi-relational graph. The circles are function nodes, the rectangles are protein nodes, the directed edges represent function assignment, the undirected edges with weights in solid line represent protein-protein interactions, and the dotted lines with weights represent function correlations. The width of the line reflects the weight of the edge.

\section{TABLE 1}

The prediction of TMC on the undirected and directed bi-relational graph for the protein nodes and function nodes, and the original function assignment.

\begin{tabular}{|c|ccc|ccc|ccc|}
\hline \multirow{2}{*}{ Nodes } & \multicolumn{4}{|c}{ original } & \multicolumn{4}{c|}{ undirected } & \multicolumn{3}{c|}{ directed } \\
\cline { 2 - 10 } & $\mathrm{f} 1$ & $\mathrm{f} 2$ & $\mathrm{f} 3$ & $\mathrm{f} 1$ & $\mathrm{f} 2$ & $\mathrm{f} 3$ & $\mathrm{f} 1$ & $\mathrm{f} 2$ & $\mathrm{f} 3$ \\
\hline p1 & 1 & 1 & 0 & 1 & 1 & 0 & 1 & 1 & 0 \\
p2 & 1 & 1 & 0 & 1 & 1 & 0 & 1 & 1 & 0 \\
p3 & 1 & 1 & 0 & 1 & 1 & 0 & 1 & 1 & 0 \\
p4 & 1 & 0 & 1 & 1 & $\mathbf{1}$ & 0 & 1 & 0 & 1 \\
p5 & 0 & 0 & 0 & 1 & $\mathbf{1}$ & 0 & 1 & 0 & 1 \\
p6 & 0 & 0 & 0 & 1 & 1 & 0 & 1 & 1 & 0 \\
\hline f1 & 1 & 0 & 0 & 1 & 0 & 0 & 1 & 0 & 0 \\
f2 & 0 & 1 & 0 & 0 & 1 & 0 & 0 & 1 & 0 \\
f3 & 0 & 0 & 1 & $\mathbf{1}$ & 0 & 0 & 0 & 0 & 1 \\
\hline
\end{tabular}

dominated by the information of $f 1$ (see the last row of Table 1), resulting in the function override problem, which was pointed out and analyzed in the previous subsection. In contrast, by using the directed bi-relational graph, these problems are avoided. The advantage of the directed bi-relational graph with respect to the undirected one will be further confirmed in our experiments on real world benchmarks.

Note, for the experiment in Figure 1 and Table 1 . we compute the function similarities using Eq. (1). Since there is no prior information on how to balance the importance of the protein and function nodes, we use the default settings of $\beta=0.5$ and $\gamma=0.5$ as in prior work [15]. For the experiment here, we can not select the best $\beta$ and $\gamma$ by grid search, since there is very scarce training data. In addition, setting higher values of $\beta$ and $\gamma$ lead to the observed problems of undirected bi-relational graphs. We convert the predicted likelihoods $F$ into binary labels using the Top $k$ scheme [18], [21], [39]. For each protein, the $k$ largest predicted probabilities are chosen as relevant functions and labeled as 1s, and the others are set as irrelevant functions and labeled as 0s. In the toy example, since each training protein has 2 functions, we set $k=2$. The other parameter settings for TMC will be detailed in the Experimental Section 4

\subsection{Transductive Multi-label Ensemble Classifica- tion}

TMC avoids the risk of overwriting the information given by function nodes. However, because of noisy edges (i.e., false positive interactions) and isolated proteins present in a single bi-relational graph, it is still limited in providing a confident likelihood score vector $F(j)$ from a single data source. To avoid this limitation, we can leverage the various graphs (or kernels) associated to the same set of proteins (e.g., PPI network, gene interaction network, and coparticipation network in a protein complex) [4], [13]. These graphs are, to some extent, independent to one another, and also carry complementary information.

Here we predict protein functions using multiple kernels derived from multiple sources by performing classifier integration. More specifically, we first transform each kernel into a directed bi-relational graph. We then train a TMC on each of these graphs. Finally, we combine these TMCs into TMEC using an ensemble approach. TMEC is described in Algorithm 1. In Eq. (15), we combine the $F_{r}$ values using a weighted majority vote. This is due to the fact that different kernels have different qualities (c.f. Figure 2 and Figure 3), and have different levels of confidence on the predicted functions of a protein. For example, if kernel $K_{1}$ is more confident on annotating protein $i$ with function $m$, and $K_{2}$ is more confident on annotating protein $i$ with function $n$, then $K_{1}$ will have more influence on determining the $m$-th function of protein $i$, and $K_{2}$ will have more influence on determining the $n$-th function of protein $i$. On the other hand, if unlabeled protein $i$ in $K_{1}$ is isolated (TMC on $K_{1}$ can not predict protein $i$ ), but it is connected with some proteins in $K_{2}$, then the functions of protein $i$ can be predicted by TMC on $K_{2}$.

Due to the different structures across different kernels, the base classifiers $F_{r}$ in Eq. (15) are diverse. In addition, because of the complementary information between different kernels, the predicted likelihoods $F_{r}(j)$ are also complementary to each other. In contrast, the annotator trained on the composite graph cannot make use of these predicted likelihoods. In ensemble learning, diversity between base classifiers is paramount to gain a consensus classifier with a good generalization ability [40]. For these reasons, 
Algorithm 1 TMEC: Transductive Multi-label Ensemble Classification

Input:

$\left\{K_{r}\right\}_{r=1}^{R}$ from $R$ data sources

$Y=\left[\mathbf{y}_{1}, \mathbf{y}_{2}, \ldots, \mathbf{y}_{l}\right]$

$\alpha, \beta, \gamma$

Output:

Predicted likelihood score vectors $\{F(j)\}_{j=l+1}^{N}$

1: Specify $\tilde{Y}$ using Eq. 6 ,

2: for $r=1$ to $R$ do

3: Set $W_{P P}=K_{r}$ and construct a directed birelational graph using Eqs.(3/4) and Eq.(10)

4: $\quad$ Get the $r$-th annotator $F_{r}$ using Eq.(14)

5: end for

6: Integrate the $R$ annotators $\left\{F_{r}\right\}_{r=1}^{R}$ into an ensemble classifier as:

$$
F(j)=\frac{1}{R} \sum_{r=1}^{R} F_{r}(j)
$$

TMEC can annotate proteins with higher confidence than approaches trained on the composite kernel and single kernels. Our experimental results in Section 5 confirm this advantage. An additional merit of TMEC is that it does not require to have all the data sources available beforehand, and each TMC can be trained individually or incrementally. Thus, new data sources can be appended into TMEC without repeating the entire training process.

\section{Experimental Setup}

\subsection{Dataset Description}

We evaluate the performance of our algorithms on three previously defined protein function prediction benchmarks, namely Yeast, Human and Fly. All these benchmarks were downloaded from the study by Mostafavi et al. $\sqrt{9]^{4}}$ and annotated according to the biological process function categories in the GO database [1]. The Yeast benchmark includes 3904 proteins annotated with 1188 functions. There are 44 different kernel functions in Yeast dataset, most of which are protein-protein interactions obtained from different experiments. The Human benchmark includes 13281 proteins annotated with 1952 GO functions. There are 8 different kernel functions in the Human dataset, which are derived from various domains, i.e., protein-protein interactions, tissue expression, proteinDNA/RNA-interaction. The Fly benchmark includes 13562 proteins annotated with 2195 GO functions. There are 38 different kernel functions in the Fly dataset, including gene expression, protein-protein interactions, and Pfam.

4. http://morrislab.med.utoronto.ca/ sara/SW/
TABLE 2

Protein function prediction benchmarks statistics $(A v g \pm$ Std means average number of functions for each protein and its standard deviation)

\begin{tabular}{|l||c|c|c|c|}
\hline Dataset & \#Kernels & \#Proteins & \#Functions & Avg \pm Std \\
\hline Yeast & 44 & 1809 & 57 & $4.35 \pm 3.28$ \\
Human & 8 & 3704 & 254 & $3.73 \pm 3.67$ \\
Fly & 38 & 3509 & 426 & $6.53 \pm 7.47$ \\
\hline
\end{tabular}

To avoid too general or too small functions, as done in previous studies [9], [18], we filtered the proteins in Yeast to include only those GO functions that had at least 100 proteins and at most 300 proteins. After this preprocessing, there are 1809 proteins annotated with 57 functions in Yeast. We filtered the proteins in Human and Fly to include only those GO functions that had at least 30 proteins and at most 100 proteins. Thus, there are 3704 proteins annotated with 254 functions in Human, and 3509 proteins annotated with 426 functions in Fly. The statistics of these filtered benchmarks are listed in the Table 2

\subsection{Evaluation Metrics}

We evaluate the protein function prediction problem as a multi-label classification problem. There are various evaluation metrics in multi-label learning [24], here we adopt three evaluation metrics, namely, Ranking Loss, Coverage and adapted Area Under the Curve (AUC). Given $C$ different protein functions, our approach results in a predicted likelihood vector that assigns a protein $i$ to a function $c$ with probability $F(i, c)$. There is no standard rule to transform the predicted likelihood vector into a binary indication label vector [24], [41]. Thus we do not apply the evaluation metrics that depend on transforming the predicted likelihood vector into a binary label vector. For brevity, the definition of Ranking Loss and Coverage is introduced in the supplementary file.

The adapted Area Under the Curve (AUC) for multilabel learning was utilized in [42]. AUC first ranks all the labels for each test instance in the descending order of their scores; it then varies the number of predicted labels from 1 to the total number of labels, and computes the receiver operating characteristic curve by calculating the true positive rate and the false positive rate for each number of predicted labels. It finally computes the area under the curve of all labels to evaluate the multi-label learning methods.

For maintaining consistency with $A U C$, we use 1-RankingLoss. Thus, the higher the value of 1RankingLoss and AUC, the better the performance. In contrast, the lower the value of Coverage, the better the performance.

\section{EXPERIMENT ANALYSIS}

We evaluate TMC and TMEC by comparing them against PfunBG [15], GRF [18], SW [9], MKL-Sum 
[19] and MKL-SA [20]. PfunBG and GRF are recently proposed semi-supervised multi-label classifiers based on PPI networks for protein function prediction. SW is a recently introduced efficient protein function prediction method based on the composite kernel. MKLSum and MKL-SA are two multi-label multiple kernel learning approaches, whose performance is verified in gene function prediction. We adopted the codes of SW 5 , MKL-SA 4 , MKL-Sum7 for our experiments. We implemented PfunBG and GRF as the authors presented in the original papers, and set the parameter values as the authors reported. TMC and PfunBG have a similar objective function. The main difference between them is that TMC is trained on the directed bi-relational graph and PfunBG is trained on the undirected bi-relational graph. Here, we use the similar settings of undirected bi-relational graph in [22] and [15], and set $\alpha$ in Eq. (7) equal to 0.01, and $\beta$ and $\gamma$ in the bi-relational graph equal to 0.5 . We set equal weights for protein nodes and function nodes, since there is no prior knowledge on the relative importance between protein and function nodes. Both MKL-Sum and MKL-SA depend on the setting of soft margin parameter $\eta$ of SVM; in the experiments, $\eta$ is chosen among $\left\{10^{-4}, 10^{-3}, \cdots, 10^{3}, 10^{4}\right\}$ using 5 -fold cross validation.

TMC, PfunBG and GRF work on a single graph (or kernel). As the authors reported in [6] and [10], the composite kernel combined with different weights has similar performance to the composite kernel combined with equal weights. Therefore, in the following experiments, TMC, PfunBG and GRF are all trained on the same composite kernel combined with individual kernels using equal weights. We also study TMC on the composite kernels combined with individual kernels in other ways, which will be detailed in Section 5.3. In addition, we compare TMEC with SW, MKLSum and MKL-SA, which learn different weights for different kernels. In Section 5.2, we investigate the performance of TMC on each single kernel. In the following experiments, we varied the ratio of labeled proteins from $10 \%$ to $60 \%$, and used the remaining proteins as unlabeled for testing. Unless otherwise specified, all the results are the average of 20 independent runs in each fixed labeled ratio.

\subsection{Directed Bi-relational Graph vs. Undirected Bi- relational Graph}

To investigate the difference between directed and undirected bi-relational graphs, we compare our TMC against PfunBG, GRF, SW, MKL-Sum and MKL-SA on the composite kernel of Yeast, Human and Fly. The composite kernel for TMC, PfunBG and GRF

5. http://morrislab.med.utoronto.ca/ sara/SW/

6. http://www.cse.msu.edu/ bucakser/ML-MKL-SA.rar

7. http://www.public.asu.edu/ ltang9/code/mkl-multiplelabel/ is a linear combination with equal weights, and the composite kernel for SW, MKL-Sum and MKL-SA is a linear combination with optimized weights. Table 3 reports the 1-RankingLoss, Table 4 lists the Coverage and Table 5 reports the AUC on the three benchmarks. Standard deviations are also reported. In these tables, results reported in boldface are significantly better, with significance level $p<0.05$. Particularly, for each fixed labeled ratio, we repeat independent experiments for each method 20 times and record the results. Then, we apply a pairwise $t$-test to check the significance of the difference among these comparative methods.

From these tables (Table 3. Table 5), we can observe that TMC trained on the directed bi-relational graph almost always performs better than PfunBG trained on the undirected bi-relational graph. These results corroborate the advantage of the directed bi-relational graph with respect to the undirected one. GRF extends the function assignment on the annotated proteins and then makes use of a semi-supervised classifier on the graph (only consisting of proteins, one protein corresponding to one node) to infer protein functions. From these tables (Table 3- Table 5), we can also see that TMC often outperforms GRF. This observation indicates that the directed bi-relational graph (consisting of protein and function nodes) is also more effective than the sole protein graph.

TMC often outperforms the other comparing methods, some of which (i.e. SW, MKL-Sum and MKLSA) are trained on the optimized composite kernel. SW takes advantage of regression to seek the optimal combining weights, and graph-based classification to predict protein functions; it achieves higher AUC on Human benchmark, but it loses to TMC on Yeast and Fly benchmarks with respect to all the evaluation metrics. Both MKL-Sum and MKL-SA are based on the optimized composite kernel and SVM to predict protein functions, but they are always outperformed by TMC. These results indicate that our equal combination of single kernels (derived from various data sources) is reasonable. The possible explanations are that the composite kernel used in TMC captures complementary information spread in different kernels [6] and multi-label classification is more suitable for protein function prediction than binary classification methods.

\subsection{Multiple Kernels vs. Single Kernel}

In this subsection, we conduct experiments on these benchmarks to investigate the advantage of classifier ensembles. We compare TMEC, GRF-MK and PfunBGMK against SW and TMC. Among the five comparing approaches, the first three are classifier integration methods, and the last two are kernel integration methods. In the experiments, the base classifiers of PfunBG-MK and GRF-MK on the individual kernels are combined in the same way as TMEC in Eq. (15). For brevity, we just report 1-RankingLoss and Coverage 
TABLE 3

(1-RankingLoss) ${ }^{*} 100$ (avg \pm std) on Yeast (44 kernels), Human (8 kernels) and Fly (38 kernels) on the composite kernel. The numbers in boldface denote the best performance (significance is examined by pairwise $t$-test with significance level $p<0.05)$.

\begin{tabular}{|l|l||c|c|c|c|c|c|}
\hline \multirow{2}{*}{ DataSet } & \multirow{2}{*}{ Method } & \multicolumn{7}{|c|}{ Labeled Ratio } \\
\cline { 3 - 7 } Yeast & 10\% & $20 \%$ & $30 \%$ & $40 \%$ & $50 \%$ & $60 \%$ \\
\hline & SW & $50.63 \pm 2.17$ & $55.58 \pm 2.37$ & $60.36 \pm 3.38$ & $64.74 \pm 1.75$ & $67.41 \pm 2.42$ & $69.90 \pm 1.95$ \\
& MKL-Sum & $53.23 \pm 3.01$ & $54.97 \pm 3.54$ & $54.46 \pm 4.09$ & $58.53 \pm 2.63$ & $61.31 \pm 9.76$ & $67.83 \pm 2.16$ \\
& MKL-SA & $62.34 \pm 0.77$ & $65.40 \pm 0.95$ & $67.87 \pm 0.88$ & $69.14 \pm 0.64$ & $70.61 \pm 0.50$ & $71.42 \pm 0.83$ \\
& GRF & $48.94 \pm 1.48$ & $54.94 \pm 1.62$ & $60.68 \pm 1.98$ & $66.12 \pm 1.18$ & $69.08 \pm 1.36$ & $71.51 \pm 1.52$ \\
& PfunBG & $65.17 \pm 1.56$ & $67.65 \pm 1.42$ & $68.46 \pm 1.28$ & $69.84 \pm 1.00$ & $68.97 \pm 1.36$ & $67.79 \pm 1.83$ \\
& TMC & $\mathbf{6 8 . 6 9} \pm \mathbf{1 . 2 4}$ & $\mathbf{7 2 . 5 8} \pm \mathbf{1 . 0 7}$ & $\mathbf{7 4 . 7 8} \pm \mathbf{0 . 7 8}$ & $\mathbf{7 6 . 9 8} \pm \mathbf{0 . 6 6}$ & $\mathbf{7 7 . 8 3} \pm \mathbf{0 . 7 5}$ & $\mathbf{7 8 . 6 0} \pm \mathbf{0 . 8 2}$ \\
\hline \multirow{5}{*}{ Humanyyyyy} & SW & $53.11 \pm 1.10$ & $63.24 \pm 1.68$ & $69.29 \pm 0.94$ & $73.99 \pm 0.73$ & $\mathbf{7 6 . 7 5} \pm \mathbf{0 . 4 8}$ & $\mathbf{7 8 . 4 9} \pm \mathbf{0 . 5 8}$ \\
& MKL-Sum & $52.11 \pm 5.58$ & $58.77 \pm 7.69$ & $53.46 \pm 5.73$ & $47.22 \pm 0.67$ & $46.19 \pm 7.71$ & $56.77 \pm 0.89$ \\
& MKL-SA & $52.30 \pm 1.93$ & $55.55 \pm 0.88$ & $55.57 \pm 0.39$ & $56.79 \pm 0.15$ & $57.13 \pm 0.47$ & $56.40 \pm 0.44$ \\
& GRF & $55.56 \pm 0.90$ & $63.21 \pm 1.27$ & $66.95 \pm 0.90$ & $69.96 \pm 0.64$ & $71.40 \pm 0.62$ & $72.31 \pm 0.42$ \\
& PfunBG & $64.60 \pm 0.86$ & $68.25 \pm 0.82$ & $69.87 \pm 0.74$ & $71.48 \pm 0.53$ & $71.93 \pm 0.70$ & $72.15 \pm 0.50$ \\
& TMC & $\mathbf{6 7 . 3 1} \pm \mathbf{0 . 7 6}$ & $\mathbf{7 1 . 5 5} \pm \mathbf{0 . 7 0}$ & $\mathbf{7 3 . 4 3} \pm \mathbf{0 . 5 6}$ & $\mathbf{7 5 . 0 3} \pm \mathbf{0 . 3 7}$ & $75.79 \pm 0.44$ & $76.25 \pm 0.49$ \\
\hline \multirow{5}{*}{ Fly } & SW & $43.81 \pm 0.78$ & $52.92 \pm 0.86$ & $61.47 \pm 0.97$ & $67.07 \pm 1.11$ & $70.37 \pm 0.87$ & $72.99 \pm 0.66$ \\
& MKL-Sum & $40.49 \pm 0.14$ & $58.57 \pm 1.95$ & $63.66 \pm 1.19$ & $65.08 \pm 1.49$ & $65.97 \pm 0.58$ & $66.54 \pm 1.23$ \\
& MKL-SA & $63.41 \pm 1.13$ & $65.37 \pm 0.26$ & $66.78 \pm 0.70$ & $68.16 \pm 0.55$ & $69.13 \pm 0.31$ & $69.80 \pm 1.14$ \\
& GRF & $44.59 \pm 0.77$ & $54.21 \pm 0.74$ & $61.54 \pm 0.72$ & $66.90 \pm 1.11$ & $70.17 \pm 0.91$ & $73.08 \pm 0.73$ \\
& PfunBG & $61.76 \pm 1.30$ & $69.22 \pm 0.64$ & $72.20 \pm 0.45$ & $74.10 \pm 0.79$ & $74.98 \pm 0.69$ & $75.67 \pm 0.57$ \\
& TMC & $\mathbf{6 4 . 9 2} \pm \mathbf{1 . 4 1}$ & $\mathbf{7 3 . 1 0} \pm \mathbf{0 . 4 8}$ & $\mathbf{7 6 . 3 9} \pm \mathbf{0 . 4 3}$ & $\mathbf{7 8 . 4 3} \pm \mathbf{0 . 4 9}$ & $\mathbf{7 9 . 7 4} \pm \mathbf{0 . 5 6}$ & $\mathbf{8 0 . 6 9} \pm \mathbf{0 . 3 7}$ \\
\hline
\end{tabular}

TABLE 4

Coverage (avg \pm std) on Yeast (44 kernels), Human (8 kernels) and Fly (38 kernels) on the composite kernel. The numbers in boldface denote the best performance (significance is examined by pairwise $t$-test with significance level $p<0.05$ ). The lower the Coverage, the better the performance.

\begin{tabular}{|c|c|c|c|c|c|c|c|}
\hline \multirow{2}{*}{ DataSet } & \multirow{2}{*}{ Method } & \multicolumn{6}{|c|}{ Labeled Ratio } \\
\hline & & $10 \%$ & $20 \%$ & $30 \%$ & $40 \%$ & $50 \%$ & $60 \%$ \\
\hline \multirow{6}{*}{ Yeast } & SW & $37.43 \pm 1.14$ & $35.38 \pm 1.06$ & $33.06 \pm 1.59$ & $30.43 \pm 0.86$ & $29.18 \pm 1.13$ & $27.40 \pm 1.06$ \\
\hline & MKL-Sum & $34.46 \pm 1.26$ & $34.60 \pm 1.41$ & $34.54 \pm 2.61$ & $32.64 \pm 1.30$ & $30.61 \pm 5.08$ & $27.08 \pm 1.46$ \\
\hline & MKL-SA & $29.80 \pm 0.43$ & $28.42 \pm 0.62$ & $26.64 \pm 0.60$ & $26.09 \pm 0.34$ & $25.18 \pm 0.53$ & $25.21 \pm 0.78$ \\
\hline & GRF & $36.69 \pm 0.95$ & $33.45 \pm 0.77$ & $30.49 \pm 0.85$ & $27.17 \pm 0.67$ & $25.37 \pm 0.78$ & $23.82 \pm 0.79$ \\
\hline & PfunBG & $27.45 \pm 1.10$ & $26.02 \pm 0.83$ & $25.67 \pm 0.67$ & $24.91 \pm 0.68$ & $25.59 \pm 0.77$ & $26.25 \pm 0.84$ \\
\hline & TMC & $25.05 \pm 0.89$ & $22.61 \pm 0.67$ & $21.20 \pm 0.52$ & $19.80 \pm 0.47$ & $19.37 \pm 0.44$ & $18.81 \pm 0.57$ \\
\hline \multirow{6}{*}{ Human } & SW & $150.52 \pm 2.75$ & $127.37 \pm 3.97$ & $111.05 \pm 2.37$ & $98.65 \pm 1.73$ & $89.83 \pm 1.65$ & $83.76 \pm 2.12$ \\
\hline & MKL-Sum & $151.68 \exists 3.21$ & $135.51 \pm 7.90$ & $149.06 \pm 15.80$ & $165.52 \pm 27.95$ & 168.12 \# 9.65 & $140.99 \pm 26.82$ \\
\hline & MKL-SA & $147.51 \pm 6.24$ & $140.41 \pm 2.20$ & $142.09 \pm 1.29$ & $139.49 \pm 0.92$ & $139.97 \pm 2.22$ & $143.25 \pm 1.80$ \\
\hline & GRF & $137.61 \pm 2.32$ & $116.65 \pm 3.36$ & $105.76 \pm 2.43$ & $97.36 \pm 1.99$ & $92.50 \pm 1.81$ & $89.35 \pm 1.53$ \\
\hline & PfunBG & $111.74 \pm 2.40$ & $101.76 \pm 2.29$ & $97.48 \pm 2.03$ & $93.87 \pm 1.64$ & $92.74 \pm 2.02$ & $92.50 \pm 1.72$ \\
\hline & TMC & $105.48 \pm 2.22$ & $93.32 \pm 2.07$ & $87.50 \pm 1.58$ & $83.20 \pm 1.35$ & $80.48 \pm 1.47$ & $78.73 \pm 1.64$ \\
\hline \multirow{6}{*}{ Fly } & SW & $307.65 \pm 2.82$ & $278.59 \pm 2.64$ & $246.06 \pm 3.21$ & $222.72 \pm 4.92$ & $206.64 \pm 3.81$ & $193.92 \pm 3.14$ \\
\hline & MKL-Sum & 276.89 \# & $251.72 \pm 9.76$ & $231.04 \pm 5.57$ & $227.34 \pm 7.51$ & $223.84 \pm 3.25$ & $221.19 \pm 5.67$ \\
\hline & MKL-SA & $228.59 \pm 6.13$ & $220.05 \pm 1.41$ & $214.54 \pm 4.29$ & $209.37 \pm 2.42$ & $203.61 \pm 1.88$ & $201.02 \pm 5.94$ \\
\hline & GRF & $293.58 \pm 2.95$ & $254.63 \pm 3.26$ & $223.75 \pm 2.60$ & $200.58 \pm 5.02$ & $184.23 \pm 3.26$ & $171.40 \pm 3.42$ \\
\hline & PfunBG & $223.49 \pm 5.54$ & $189.68 \pm 3.21$ & $176.24 \pm 1.93$ & $168.82 \pm 3.64$ & $164.84 \pm 2.66$ & $163.99 \pm 3.18$ \\
\hline & TMC & $211.78 \pm 6.49$ & $173.48 \pm 2.86$ & $156.77 \pm 2.34$ & $147.41 \pm 2.87$ & $140.09 \pm 2.83$ & $135.80 \pm 2.75$ \\
\hline
\end{tabular}

on these three datasets with $30 \%$ and $60 \%$ annotated proteins in Table 6 and Table 7 . The results with respect to other labeled ratios are similar, and are excluded for brevity.

We can observe that TMEC on the multiple kernels often outperforms TMC on the composite kernel, and TMEC always performs better than SW. GRF-MK and PfunBG-MK sometimes also outperform TMC, whereas TMC outperforms GRF and PfunBG on the composite kernel. This fact demonstrates the advantage of classifier integration with respect to the kernel integration method in protein function prediction.
We conduct additional experiments (with $80 \%$ proteins annotated and $20 \%$ used for testing) to investigate the difference between the TMC on the composite kernel, TMC on a single kernel from one data source, and TMEC on multiple kernels. We show the result with respect to 1-RankingLoss and Coverage in Figure 2 and Figure 3 . In all these figures, the first two bars represent TMEC (red bar) and TMC (white bar) trained on the composite kernel; the remaining bars (grey bars) describe the results of TMC trained on a single kernel. The highest bar (1-RankingLoss) in Figure 2 and the lowest bar (Coverage) in Figure 3 indicate that the 
TABLE 5

$A U C^{*} 100$ (avg \pm std) on Yeast (44 kernels), Human (8 kernels) and Fly (38 kernels) on the composite kernel.

The numbers in boldface denote the best performance (significance is examined by pairwise $t$-test with significance level $p<0.05)$.

\begin{tabular}{|l|l||c|c|c|c|c|c|}
\hline \multirow{2}{*}{ DataSet } & \multirow{2}{*}{ Method } & \multicolumn{7}{|c|}{ Labeled Ratio } \\
\cline { 3 - 7 } & & $10 \%$ & $20 \%$ & $30 \%$ & $40 \%$ & $50 \%$ & $60 \%$ \\
\hline & SW & $54.72 \pm 0.65$ & $59.22 \pm 1.28$ & $63.08 \pm 1.53$ & $65.38 \pm 1.58$ & $67.63 \pm 1.14$ & $71.18 \pm 0.86$ \\
& MKL-Sum & $54.46 \pm 2.11$ & $57.02 \pm 2.68$ & $55.80 \pm 3.56$ & $58.46 \pm 2.49$ & $60.38 \pm 8.27$ & $66.38 \pm 2.37$ \\
Yeast & MKL-SA & $62.71 \pm 0.91$ & $64.74 \pm 0.60$ & $66.54 \pm 0.76$ & $67.80 \pm 0.81$ & $69.22 \pm 0.55$ & $70.11 \pm 1.29$ \\
& GRF & $56.29 \pm 1.48$ & $62.67 \pm 1.19$ & $68.52 \pm 1.48$ & $70.88 \pm 1.45$ & $73.41 \pm 0.70$ & $75.32 \pm 1.08$ \\
& PfunBG & $68.26 \pm 1.05$ & $71.08 \pm 0.93$ & $71.51 \pm 1.39$ & $71.90 \pm 0.60$ & $72.11 \pm 0.53$ & $72.11 \pm 0.77$ \\
& TMC & $\mathbf{6 9 . 0 2} \pm \mathbf{0 . 8 1}$ & $\mathbf{7 2 . 9 4} \pm \mathbf{0 . 9 3}$ & $\mathbf{7 4 . 2 3} \pm \mathbf{1 . 0 0}$ & $\mathbf{7 5 . 5 9} \pm \mathbf{0 . 4 1}$ & $\mathbf{7 6 . 5 3} \pm \mathbf{0 . 6 6}$ & $\mathbf{7 7 . 6 8} \pm \mathbf{0 . 6 9}$ \\
\hline \multirow{5}{*}{ Humanyyyyyy} & SW & $60.73 \pm 1.06$ & $67.91 \pm 0.70$ & $72.51 \pm 0.37$ & $75.15 \pm 0.74$ & $\mathbf{7 7 . 3 5} \pm \mathbf{0 . 5 1}$ & $\mathbf{7 8 . 7 0} \pm \mathbf{0 . 7 9}$ \\
& MKL-Sum & $51.07 \pm 6.83$ & $57.58 \pm 8.24$ & $51.71 \pm 4.80$ & $47.33 \pm 9.57$ & $46.74 \pm 7.59$ & $55.6 \pm 10.4$ \\
& MKL-SA & $53.03 \pm 1.03$ & $54.98 \pm 0.52$ & $55.33 \pm 0.33$ & $55.68 \pm 0.48$ & $55.54 \pm 0.64$ & $55.27 \pm 0.55$ \\
& GRF & $63.50 \pm 1.19$ & $68.38 \pm 0.70$ & $70.95 \pm 0.28$ & $72.69 \pm 0.56$ & $73.79 \pm 0.66$ & $74.76 \pm 0.54$ \\
& PfunBG & $68.48 \pm 0.70$ & $70.76 \pm 0.56$ & $72.50 \pm 0.50$ & $73.15 \pm 0.54$ & $73.66 \pm 0.56$ & $73.95 \pm 0.68$ \\
& TMC & $\mathbf{6 9 . 5 2} \pm \mathbf{0 . 4 3}$ & $\mathbf{7 2 . 4 8} \pm \mathbf{0 . 3 7}$ & $\mathbf{7 3 . 9 7} \pm \mathbf{0 . 3 8}$ & $74.89 \pm 0.38$ & $75.34 \pm 0.40$ & $76.36 \pm 0.44$ \\
\hline & SW & $53.39 \pm 0.69$ & $58.85 \pm 0.85$ & $63.56 \pm 0.87$ & $66.77 \pm 0.79$ & $68.17 \pm 0.75$ & $69.99 \pm 0.31$ \\
& MKL-Sum & $51.75 \pm 2.40$ & $55.96 \pm 1.24$ & $60.06 \pm 0.73$ & $60.94 \pm 1.23$ & $61.49 \pm 0.70$ & $62.40 \pm 0.98$ \\
Fly & MKL-SA & $59.81 \pm 0.66$ & $61.10 \pm 0.48$ & $62.02 \pm 0.45$ & $63.00 \pm 0.26$ & $63.84 \pm 0.54$ & $64.68 \pm 0.88$ \\
& GRF & $54.39 \pm 0.59$ & $61.44 \pm 0.80$ & $65.75 \pm 0.66$ & $69.28 \pm 0.87$ & $72.10 \pm 0.52$ & $73.62 \pm 0.66$ \\
& PfunBG & $65.67 \pm 0.67$ & $70.18 \pm 0.49$ & $71.94 \pm 0.34$ & $72.95 \pm 0.40$ & $73.77 \pm 0.52$ & $73.66 \pm 0.47$ \\
& TMC & $\mathbf{6 6 . 8 2} \pm \mathbf{0 . 5 2}$ & $\mathbf{7 1 . 6 1} \pm \mathbf{0 . 3 5}$ & $\mathbf{7 3 . 6 5} \pm \mathbf{0 . 3 0}$ & $\mathbf{7 4 . 8 7} \pm \mathbf{0 . 3 8}$ & $\mathbf{7 6 . 0 8} \pm \mathbf{0 . 6 3}$ & $\mathbf{7 6 . 4 2} \pm \mathbf{0 . 7 1}$ \\
\hline
\end{tabular}

TABLE 6

(1-RankingLoss) ${ }^{*} 100(\mathrm{avg} \pm \mathrm{std})$ on the composite kernel and multiple kernels of Yeast (44 kernels), Human (8 kernels) and Fly (38 kernels).

\begin{tabular}{|l||c|c|c|c|c|c|}
\hline \multicolumn{1}{|c||}{} & \multicolumn{2}{c|}{ Yeast } & \multicolumn{2}{c|}{ Human } & \multicolumn{2}{c|}{ Fly } \\
\cline { 2 - 7 } & $30 \%$ & $60 \%$ & $30 \%$ & $60 \%$ & $30 \%$ & $60 \%$ \\
\hline SW & $60.36 \pm 3.38$ & $69.90 \pm 1.95$ & $69.29 \pm 0.94$ & $78.49 \pm 0.58$ & $61.47 \pm 0.97$ & $72.99 \pm 0.66$ \\
TMC & $74.78 \pm 0.78$ & $78.60 \pm 0.82$ & $73.43 \pm 0.56$ & $76.25 \pm 0.49$ & $76.39 \pm 0.43$ & $80.69 \pm 0.37$ \\
GRF-MK & $68.74 \pm 1.66$ & $74.12 \pm 1.16$ & $76.02 \pm 0.71$ & $81.11 \pm 0.51$ & $68.13 \pm 0.65$ & $75.45 \pm 0.58$ \\
PfunBG-MK & $73.48 \pm 0.90$ & $75.70 \pm 1.05$ & $77.57 \pm 0.52$ & $81.60 \pm 0.50$ & $74.67 \pm 0.40$ & $78.31 \pm 0.42$ \\
TMEC & $\mathbf{7 6 . 7 5} \pm \mathbf{0 . 5 7}$ & $\mathbf{8 0 . 1 8} \pm \mathbf{0 . 6 1}$ & $\mathbf{7 9 . 3 0} \pm \mathbf{0 . 4 3}$ & $\mathbf{8 3 . 4 0} \pm \mathbf{0 . 4 6}$ & $\mathbf{7 7 . 2 4} \pm \mathbf{0 . 4 1}$ & $\mathbf{8 1 . 1 6} \pm \mathbf{0 . 3 7}$ \\
\hline
\end{tabular}

TABLE 7

Coverage (avg \pm std) on the composite kernel and multiple kernels of Yeast (44 kernels), Human (8 kernels) and Fly (38 kernels).

\begin{tabular}{|l||c|c|c|c|c|c|}
\hline \multicolumn{1}{|c|}{} & \multicolumn{2}{c|}{ Yeast } & \multicolumn{2}{c|}{ Human } & \multicolumn{2}{c|}{ Fly } \\
\cline { 2 - 7 } & $30 \%$ & $60 \%$ & $30 \%$ & $60 \%$ & $30 \%$ & $60 \%$ \\
\hline SW & $33.06 \pm 1.59$ & $27.40 \pm 1.06$ & $111.05 \pm 2.37$ & $83.76 \pm 2.12$ & $246.06 \pm 3.21$ & $193.92 \pm 3.14$ \\
TMC & $21.20 \pm 0.52$ & $18.81 \pm 0.57$ & $87.50 \pm 1.58$ & $78.73 \pm 1.64$ & $156.77 \pm 2.34$ & $135.80 \pm 2.75$ \\
GRF-MK & $25.80 \pm 0.83$ & $22.18 \pm 0.66$ & $81.39 \pm 2.00$ & $66.08 \pm 1.85$ & $194.44 \pm 2.37$ & $160.68 \pm 3.03$ \\
PfunBG-MK & $22.51 \pm 0.53$ & $21.22 \pm 0.63$ & $76.73 \pm 1.46$ & $65.63 \pm 1.80$ & $164.40 \pm 1.96$ & $149.65 \pm 2.95$ \\
TMEC & $\mathbf{1 9 . 9 4} \pm \mathbf{0 . 3 6}$ & $\mathbf{1 7 . 7 2} \pm \mathbf{0 . 5 0}$ & $\mathbf{7 1 . 8 6} \pm \mathbf{1 . 2 5}$ & $\mathbf{5 9 . 6 7} \pm \mathbf{1 . 7 2}$ & $\mathbf{1 5 1 . 6 3} \pm \mathbf{2 . 1 1}$ & $\mathbf{1 3 2 . 0 2} \pm \mathbf{2 . 9 4}$ \\
\hline
\end{tabular}

corresponding results are significantly better than the others bars.

We observe that TMC trained on the composite kernel always outperforms TMC trained on a single kernel. There are three possible reasons. First, there are some isolated proteins in each data source, whose functions can not be predicted using a single kernel derived from a single data source. Second, an isolated protein in one kernel may be connected with other proteins in other data sources. Therefore, there are few (or no) isolated proteins in the composite kernel, which includes the complementary information across different kernels. Third, the similarity between two proteins from the composite kernel is often more reliable than that from a single kernel (derived from a single data source).

TMEC performs better than TMC trained on the single kernel, and it outperforms TMC trained on the composite kernel. TMEC not only takes advantage of the complementary information between different kernels, but also makes use of the structural difference among different kernels and the complementary predicted likelihood score vectors. These results also corroborate the advantage of classifier integration versus kernel integration. 


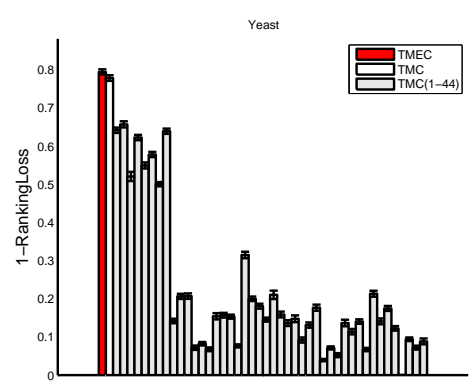

(a) Yeast

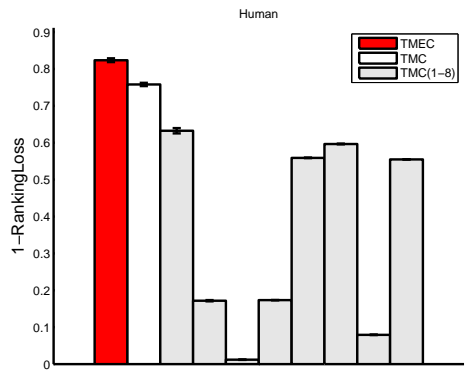

(b) Human

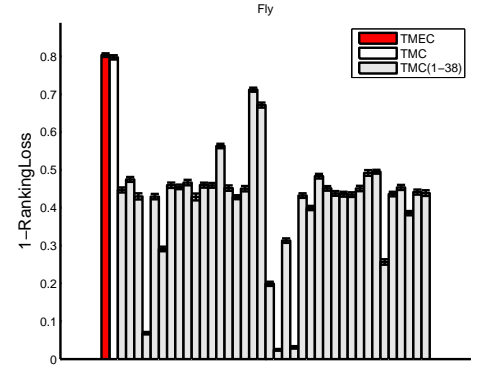

(c) Fly

Fig. 2. Difference between multiple kernels (red bar), composite kernel (white bar) and single kernels (grey bars) of Yeast, Human and Fly, with respect to (1-RankingLoss)*100.

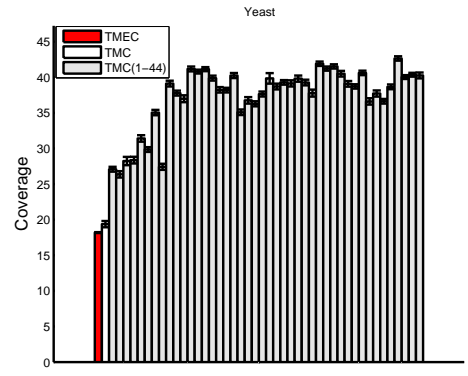

(a) Yeast

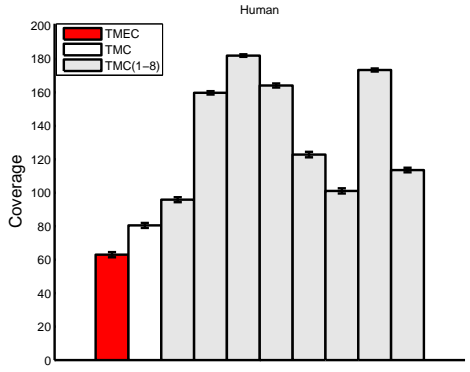

(b) Human

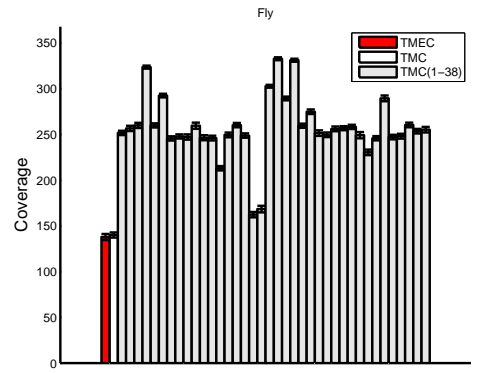

(c) Fly

Fig. 3. Difference between multiple kernels (red bar), composite kernel (white bar) and single kernels (grey bars) of Yeast, Human and Fly, with respect to Coverage.

TABLE 8

(1-RankingLoss) ${ }^{*} 100$ (avg \pm std) on Yeast (44 kernels), Human (8 kernels) and Fly (38 kernels), with respect to different weighting schemes

\begin{tabular}{|l||c|c|c|c|c|c|}
\hline \multirow{2}{*}{} & \multicolumn{2}{c|}{ Yeast } & \multicolumn{2}{c|}{ Human } & \multicolumn{2}{c|}{ Fly } \\
\cline { 2 - 7 } & $20 \%$ & $50 \%$ & $20 \%$ & $50 \%$ & $20 \%$ & $50 \%$ \\
\hline TMC-B & $69.41 \pm 1.01$ & $74.70 \pm 0.79$ & $75.08 \pm 0.68$ & $81.75 \pm 0.56$ & $70.24 \pm 0.73$ & $77.85 \pm 0.41$ \\
TMC-BW & $71.98 \pm 1.00$ & $77.12 \pm 0.80$ & $75.35 \pm 0.68$ & $82.10 \pm 0.56$ & $72.17 \pm 0.67$ & $79.70 \pm 0.45$ \\
TMC-E & $72.58 \pm 1.07$ & $78.60 \pm 0.75$ & $71.55 \pm 0.70$ & $76.25 \pm 0.44$ & $73.10 \pm 0.48$ & $80.69 \pm 0.56$ \\
TMEC & $\mathbf{7 4 . 7 1} \pm \mathbf{0 . 8 0}$ & $\mathbf{8 0 . 1 8} \pm \mathbf{0 . 6 7}$ & $\mathbf{7 6 . 8 6} \pm \mathbf{0 . 5 3}$ & $\mathbf{8 3 . 4 0} \pm \mathbf{0 . 5 0}$ & $\mathbf{7 4 . 2 9} \pm \mathbf{0 . 3 9}$ & $\mathbf{8 1 . 1 6} \pm \mathbf{0 . 5 5}$ \\
\hline
\end{tabular}

\subsection{Influence of Different Weighting Schemes}

In this section, we investigate the performance of TMC on the different composite kernels, obtained by combining individual kernels using different weighting schemes. Here, we use two approaches to combine the single kernels, plus the equal weight one already used in TMC. The first one is a binary way. We set the weight of the edge between protein $i$ and $j$ as:

$$
K_{B}(i, j)=\left\{\begin{array}{l}
1, \text { if } \sum_{r=1}^{R} K_{r}(i, j)>0 \\
0, \text { otherwise }
\end{array}\right.
$$

Thus if there is an edge between proteins $i$ and $j$ in any of the individual kernels, we specify the edge weight between them as 1 . The second one is a weighted version; we set the weight of the edge between proteins $i$ and $j$ as:

$$
K_{B W}(i, j)=\frac{\sum_{r=1}^{R} \delta\left(K_{r}(i, j)\right)}{R}
$$

where $\delta\left(K_{r}(i, j)\right)=1$ if $K_{r}(i, j)>0$, and $\delta\left(K_{r}(i, j)\right)=$ 0 otherwise. Thus $K_{B W}(i, j)$ is proportional to the number of edges between proteins $i$ and $j$ in the individual kernels. The composite kernel used in TMC is a summation of single kernels with equal weights, it is defined as:

$$
K_{E}(i, j)=\frac{\sum_{r=1}^{R} K_{r}(i, j)}{R}
$$

We then train TMC on $K_{B}, K_{B W}$ and $K_{E}$, and name the resulting classifiers as TMC-B, TMC-BW and TMCE, respectively. For brevity, we just report the results (along with the results of TMEC on multiple kernels) 
with respect to 1-RankingLoss in Table 8 with $20 \%$ and $50 \%$ proteins annotated. The results with respect to Coverage are reported in the supplementary file. The results with respect to other labeled ratios have similar observations.

From these tables, we observe that none of these three kernel integration methods performs significantly better than others, and TMC-E often works better than the other two kernel integration techniques. Another observation is that TMEC always achieves better performance than the three different kernel integration methods (TMC-B, TMC-BW and TMC-E). These results further demonstrate the advantage of classifier ensemble with respect to kernel integration.

\subsection{Influence of Different Ensemble Techniques}

In this section, we conduct experiments to explore the performance of TMEC with respect to several other ensemble techniques, namely decision templates [43] and linear regression based ensemble [44]. The detail of these two ensemble technique is introduced in the supplementary file.

We utilize decision templates, linear regression based ensemble, and Eq. (15) to integrate TMCs trained on individual kernels, and name the resulting ensemble classifiers as TMEC-DT, TMEC-Reg, and TMEC, respectively. For brevity, we just report the results with respect to 1-RankingLoss in Table 9 We report the results with respect to Coverage in the supplementary file. In the experiment, $50 \%$ of the data are randomly selected for training and the remaining $50 \%$ are used for testing. The other settings of the experiments are kept the same as described in the Experimental Setup 4.

TABLE 9

Performance ((1-RankingLoss)*100) with respect to different ensemble techniques.

\begin{tabular}{|l||c|c|c|}
\hline Methods & Yeast & Human & Fly \\
\hline TMEC & $\mathbf{7 9 . 2 1} \pm \mathbf{0 . 7 6}$ & $\mathbf{8 2 . 1 7} \pm \mathbf{0 . 4 5}$ & $\mathbf{8 0 . 5 8} \pm \mathbf{0 . 5 0}$ \\
TMEC-DT & $61.94 \pm 0.40$ & $65.07 \pm 0.41$ & $68.12 \pm 0.32$ \\
TMEC-Reg & $61.17 \pm 1.00$ & $70.30 \pm 1.08$ & $61.50 \pm 1.19$ \\
\hline
\end{tabular}

From these tables, we can observe that TMEC, which uses simple weighted majority vote as in Eq. (15) to combine base classifiers, outperforms TMEC-DT and TMEC-Reg, which use optimized weights to integrate base classifiers. This is because there were many isolated proteins in each single data source of our experimental datasets. The functions of these isolated proteins cannot be predicted by using a single data source alone. In addition, the predicted likelihoods from each single data source are not reliable. Learning from these unreliable predictions results in incorrect learned weights for each of the base classifiers, which deteriorates the ensemble classification performance.

\subsection{Parameter Sensitivity Analysis}

There are three parameters: $\alpha, \beta$ and $\gamma$ in TMC and TMEC. $\alpha$ is used to balance the tradeoff between label propagation and initial label assignment; $\alpha$ is often set to a small value (i.e., $\alpha=0.01$ ) [15], [22]. In this section, to explore the sensitivity of our methods with respect to $\beta$ and $\gamma$, we vary $\beta$ and $\gamma$ between 0.1 and 0.9 with step size 0.1 . The settings of $\beta=0,1$ and $\gamma=0,1$ are not reasonable for the directed birelational graph. If we set $\beta=0$, there is no function propagation among proteins. If we set $\beta=1$, there is no function propagation from function to protein nodes. If we set $\gamma=0$, there is no function annotations on the protein nodes in the directed bi-relational graph. If we set $\gamma=1$, there is no propagation probability between protein and function nodes. Given these reasons, we vary $\beta$ and $\gamma$ in $[0.1,0.9]$ to investigate the parameter sensitivity of TMC and TMEC. The recorded 1-RankingLoss and Coverage with respect to different settings of $\beta$ and $\gamma$ are shown in Figure 4 for the Human benchmark. The recorded 1-RankingLoss and Coverage for the Yeast benchmark are included in the supplementary file. Here, we utilize the surf figure to visualize the parameter sensitivity of TMC and TMEC with respect to $\beta$ and $\gamma$. The density plot shows that similar colors in the same figure are parameters with similar predictive performance. We also fix the scale of TMC and TMEC in the same range for each evaluation metric on each dataset.

From these figures, we can observe that TMEC is less sensitive than TMC to parameter selection. TMEC has wider ranges of effective parameter values than TMC, and it can often achieve better performance than TMC in the same parameters setting. These advantages can be attributed to the fact that TMEC not only makes use of the structure differences among the single kernels from various data sources, but also takes advantage of the complimentary information among the base classifiers on each single kernel. These results again support the advantage of classifier integration over kernel integration.

Both TMC and TMEC are more sensitive to $\gamma$ than to $\beta$. $\gamma$ adjusts the distribution of function labels on protein and function nodes. $\beta$ adjusts the importance of the inter-subgraph (between proteins and functions) and intra-subgraph (between pairwise proteins, or pairwise functions), it determines the speed of label information propagated from function nodes to protein nodes. TMEC achieved relative stable performance when $\gamma \in[0.4,0.9]$ and TMC reaches relative stable performance when $\gamma \in[0.5,0.9]$. Bigger $\gamma$ means more emphasis on the initial function assignment. This fact reinforces that it is important to keep the original label assignment in label propagation, it also indicates the rationality of the proposed directed bi-relational graph. 


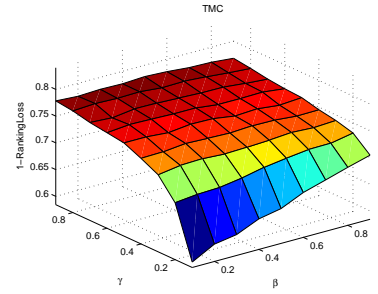

(a) TMC (1-RankingLoss)*100

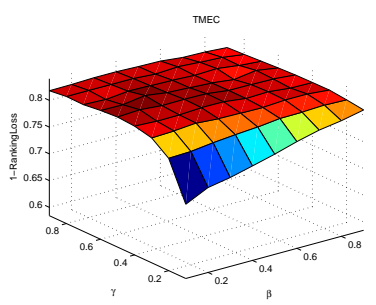

(b) TMEC (1-RankingLoss)*100

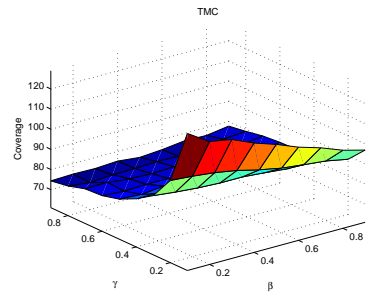

(c) TMC (Coverage)

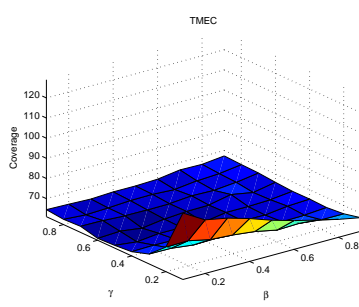

(d) TMEC(Coverage)

Fig. 4. 1-RankingLoss and Coverage on different $\beta$ and $\gamma$ (Human). Similar colors in the figure are parameters with similar predictive performance.

\section{Conclusions}

In this paper, we analyze the drawback of using undirected bi-relational graphs in label propagation. To avoid this limitation, we propose to use a directed bi-relational graph, and define a TMC on it. We further improve the performance by combining various TMCs trained on multiple data sources (TMEC). Different from traditional methods that make use of multiple data sources by kernel integration, TMEC takes advantage of multiple data sources by classifier integration. TMEC does not require to collect all the data sources beforehand. Our experimental results show that classifier integration is a valuable methodology to leverage multiple biological data sources.

\section{ACKNOWLEDGEMENT}

We are thankful to the anonymous reviewers and editors for their valuable comments. This paper is partially supported by grants from NSF IIS-0905117, NSF Career Award IIS-1252318, Natural Science Foundation of China (Project Nos. 61070090, 61003174, 61101234), Natural Science Foundation of Guangdong Province (S2012010009961), Specialized Research Fund for the Doctoral Program of Higher Education (20110172120027), Fundamental Research Funds for the Central Universities of China (XDJK2010B002) and China Scholarship Council (CSC).

\section{REFERENCES}

[1] G. O. Consortium et al., "Gene ontology: tool for the unification of biology," Nature Genetics, vol. 25, no. 1, pp. 25-29, 2000.

[2] R. Sharan, I. Ulitsky, and R. Shamir, "Network-based prediction of protein function," Molecular Systems Biology, vol. 3, no. 1, 2007.

[3] G. Pandey, V. Kumar, and M. Steinbach, "Computational approaches for protein function prediction," Department of Computer Science and Engineering, University of Minnesota, Twin Cities, Tech. Rep. TR 06-028, 2006.

[4] W. Noble and A. Ben-Hur, "Integrating information for protein function prediction," Bioinformatics-From Genomes to Therapies, pp. 1297-1314, 2007.

[5] G. Lanckriet, T. De Bie, N. Cristianini, M. Jordan, and W. Noble, "A statistical framework for genomic data fusion," Bioinformatics, vol. 20, no. 16, pp. 2626-2635, 2004.

[6] D. Lewis, "Combining kernels for classification," Ph.D. dissertation, Columbia University, 2006.
[7] C. Leslie, E. Eskin, A. Cohen, J. Weston, and W. Noble, "Mismatch string kernels for discriminative protein classification," Bioinformatics, vol. 20, no. 4, pp. 467-476, 2004.

[8] P. Pavlidis, J. Weston, J. Cai, and W. Noble, "Learning gene functional classifications from multiple data types," Journal of Computational Biology, vol. 9, no. 2, pp. 401-411, 2002.

[9] S. Mostafavi and Q. Morris, "Fast integration of heterogeneous data sources for predicting gene function with limited annotation," Bioinformatics, vol. 26, no. 14, pp. 1759-1765, 2010.

[10] K. Tsuda, H. Shin, and B. Schölkopf, "Fast protein classification with multiple networks," Bioinformatics, vol. 21, no. suppl 2, p ii59, 2005.

[11] M. Re and G. Valentini, "Ensemble based data fusion for gene function prediction," Multiple Classifier Systems, pp. 448-457, 2009.

[12] O. Chapelle, B. Schölkopf, A. Zien et al., Semi-supervised learning. MIT press Cambridge, 2006, vol. 2.

[13] H. Shin, K. Tsuda, and B. Schölkopf, "Protein functional class prediction with a combined graph," Expert Systems with Applications, vol. 36, no. 2, pp. 3284-3292, 2009.

[14] J. Weston, C. Leslie, E. Ie, D. Zhou, A. Elisseeff, and W. Noble, "Semi-supervised protein classification using cluster kernels," Bioinformatics, vol. 21, no. 15, pp. 3241-3247, 2005.

[15] J. Jiang, "Learning protein functions from bi-relational graph of proteins and function annotations," Algorithms in Bioinformatics, pp. 128-138, 2011.

[16] J. Jiang and L. McQuay, "Predicting protein function by multi-label correlated semi-supervised learning," IEEE/ACM Transactions on Computational Biology and Bioinformatics, vol. 9, no. 4, pp. 1059-1069, 2012.

[17] G. Pandey, C. Myers, and V. Kumar, "Incorporating functional inter-relationships into protein function prediction algorithms," BMC Bioinformatics, vol. 10, no. 1, p. 142, 2009.

[18] X. Zhang and D. Dai, "A framework for incorporating functional interrelationships into protein function prediction algorithms," IEEE/ACM Transactions on Computational Biology and Bioinformatics, vol. 9, no. 3, pp. 740-753, 2012.

[19] L. Tang, J. Chen, and J. Ye, "On multiple kernel learning with multiple labels," in Proceedings of 21st International Jont Conference on Artifical Intelligence(IJCAI), 2009, pp. 1255-1260.

[20] S. Bucak, R. Jin, and A. Jain, "Multi-label multiple kernel learning by stochastic approximation: Application to visual object recognition," in Proceedings of Advances in Neural Information Processing Systems (NIPS), 2010, pp. 1145-1154.

[21] G. Yu, C. Domeniconi, H. Rangwala, G. Zhang, and Z. Yu, "Transductive multi-label ensemble classification for protein function prediction," in Proceedings of the 18th ACM SIGKDD International Conference on Knowledge Discovery and Data Mining (SIGKDD), 2012, pp. 1077-1085.

[22] H. Wang, H. Huang, and C. Ding, "Image annotation using birelational graph of images and semantic labels," in Proceedings of IEEE Conference on Computer Vision and Pattern Recognition (CVPR), 2011, pp. 793-800.

[23] A. Ruepp, A. Zollner, D. Maier, K. Albermann, J. Hani, M. Mokrejs, I. Tetko, U. Güldener, G. Mannhaupt, M. Münsterkötter et al., "The funcat, a functional annotation scheme for systematic classification of proteins from whole genomes," Nucleic Acids Research, vol. 32, no. 18, pp. 5539-5545, 2004. 
[24] G. Tsoumakas, I. Katakis, and I. Vlahavas, "Mining multi-label data," Data Mining and Knowledge Discovery Handbook, pp. 667$685,2010$.

[25] A. Elisseeff and J. Weston, "A kernel method for multi-labelled classification," in Proceedings of Advances in Neural Information Processing Systems (NIPS), 2001, pp. 681-687.

[26] G. Chen, J. Zhang, F. Wang, C. Zhang, and Y. Gao, "Efficient multi-label classification with hypergraph regularization," in Proceedings of IEEE Conference on Computer Vision and Pattern Recognition, 2009, pp. 1658-1665.

[27] Z. Barutcuoglu, R. Schapire, and O. Troyanskaya, "Hierarchical multi-label prediction of gene function," Bioinformatics, vol. 22, no. 7 , pp. $830-836,2006$.

[28] G. Valentini, "True path rule hierarchical ensembles for genomewide gene function prediction," IEEE/ACM Transactions on Computational Biology and Bioinformatics, vol. 8, no. 3, pp. 832847, 2011.

[29] D. Lin, "An information-theoretic definition of similarity," in Proceedings of the 15th International Conference on Machine Learning, 1998, pp. 296-304.

[30] D. Zhou, O. Bousquet, T. Lal, J. Weston, and B. Schölkopf, "Learning with local and global consistency," in Proceedings of Advances in Neural Information Processing Systems (NIPS), 2004, pp. 321-328

[31] M. Belkin, P. Niyogi, and V. Sindhwani, "Manifold regularization: a geometric framework for learning from labeled and unlabeled examples," Journal of Machine Learning Research, vol. 7, pp. 2399-2434, 2006.

[32] A. Dempster, N. Laird, and D. Rubin, "Maximum likelihood from incomplete data via the em algorithm," Journal of the Royal Statistical Society. Series B (Methodological), pp. 1-38, 1977.

[33] S. Mostafavi, D. Ray, D. Warde-Farley, C. Grouios, and Q. Morris, "Genemania: a real-time multiple association network integration algorithm for predicting gene function," Genome Biology, vol. 9, no. Suppl 1, p. S4, 2008.

[34] N. Cesa-Bianchi, M. Re, and G. Valentini, "Synergy of multilabel hierarchical ensembles, data fusion, and cost-sensitive methods for gene functional inference," Machine Learning, vol. 88, no. 1-2, pp. 1-33, 2012.

[35] M. Gönen and E. Alpaydin, "Multiple kernel learning algorithms," Journal of Machine Learning Research, vol. 12, pp. 2211$2268,2011$.

[36] Z. Zha, T. Mei, J. Wang, Z. Wang, and X. Hua, "Graph-based semi-supervised learning with multiple labels," Journal of Visual Communication and Image Representation, vol. 20, no. 2, pp. 97103, 2009.

[37] H. Tong, C. Faloutsos, and J. Pan, "Random walk with restart: fast solutions and applications," Knowledge and Information Systems, vol. 14, no. 3, pp. 327-346, 2008.

[38] B. Schwikowski, P. Uetz, S. Fields et al., "A network of proteinprotein interactions in yeast," Nature Biotechnology, vol. 18, no. 12, pp. 1257-1261, 2000.

[39] P. Bogdanov and A. Singh, "Molecular function prediction using neighborhood features," IEEE/ACM Transactions on Computational Biology and Bioinformatics, vol. 7, no. 2, pp. 208-217, 2010.

[40] L. Kuncheva and C. Whitaker, "Measures of diversity in classifier ensembles and their relationship with the ensemble accuracy," Machine Learning, vol. 51, no. 2, pp. 181-207, 2003.

[41] R. Fan and C. Lin, "A study on threshold selection for multilabel classification," Department of Computer Science, National Taiwan University, Tech. Rep., 2007.

[42] S. Bucak, R. Jin, and A. Jain, "Multi-label learning with incomplete class assignments," in Proceedings of IEEE Conference on Computer Vision and Pattern Recognition (CVPR), 2011, pp. 2801-2808.

[43] L. I. Kuncheva, J. C. Bezdek, and R. Duin, "Decision templates for multiple classifier fusion: an experimental comparison," Pattern Recognition, vol. 34, no. 2, pp. 299-314, 2001.

[44] G. Yu, G. Zhang, Z. Zhang, Z. Yu, and L. Deng, "Semisupervised classification based on subspace sparse representation," Knowledge and Information Systems, Minor Revision and Resubmitted on 2013-06-29.

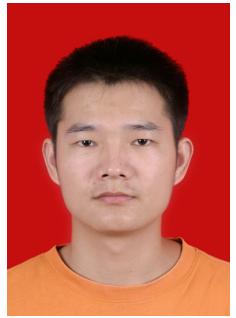

Guoxian $\mathrm{Yu}$ is an Associate Professor in the College of Computer and Information Science, Southwest University, Chongqing, China. He received B.Sc. degree in Software Engineering from Xi'an University of Technology, Xi'an, China in 2007, and Ph.D. in Computer Science from South China University of Technology, Guangzhou, China in 2013. He visited the Data Mining Lab in the George Mason University, VA, USA from 2011 to 2013. His current research interests include machine learning, data mining and bioinformatics. He is a recipient of Best Poster Award of SDM12 Doctral Forum and Best Student Paper Award of 10th IEEE International Conference on Machine Learning and Cybernetics (ICMLC).

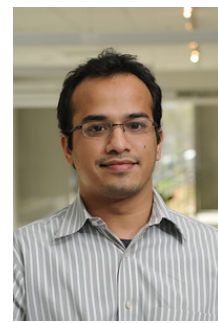

Huzefa Rangwala is an Assistant Profes sor at the department of Computer Science, George Mason University, VA, USA. He received his Ph.D. in Computer Science from the University of Minnesota in the year 2008. His core research interests include bioinformatics, machine learning, and high performance computing. He is the recipient of the NSF Early Faculty Career Award in 2013, the 2013 Volgenau Outstanding Teaching Faculty Award, 2012 Computer Science Department Outstanding Teaching Faculty Award and 2011 Computer Science Department Outstanding Junior Researcher Award.

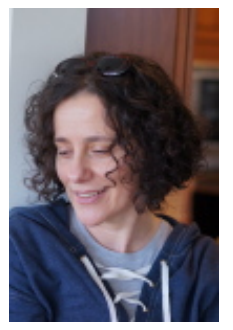

Carlotta Domeniconi is an Associate Professor in the Department of Computer Science at George Mason University, VA, USA. She received a B.Sc. degree in Computer Science from the University of Milan, Italy, in 1992, and a Ph.D. degree in Computer Science from the University of California, Riverside, in 2002. Her research interests include pattern recognition, machine learning, data mining, and feature relevance estimation. She has published extensively in premier data mining and machine learning conferences and journals. She has served as PC member for KDD, ICDM, SDM, ECML-PKDD, and AAAI, and she is an Associate Editor of the IEEE Transactions of Neural Networks and Learning Systems. Her research is in part supported NSF CAREER Award, US Army, the Air Force, and the DoD.

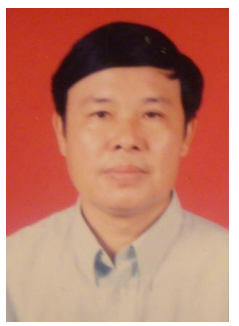

Guoji Zhang is a Professor at the School of Sciences, South China University of Technology, Guangzhou, China. He received his B.Sc. degree in Computer Application and Ph.D. degree in Circuit and System from South China University of Technology, in 1977 and 1999, respectively. His research interests include computational intelligence, computational electromagnetic and cryptology, he has published over 50 research papers.

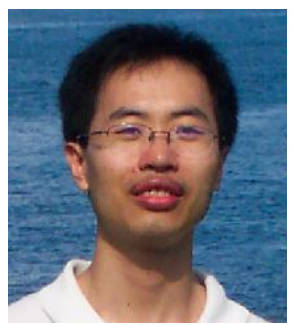

Zhiwen Yu is a Professor in the School of Computer Science and Engineering, South China University of Technology, Guangzhou, China. He received the B.Sc. and M.Phil. degrees from the Sun Yat-Sen University in China in 2001 and 2004 respectively, and the Ph.D. degree in Computer Science from the City University of Hong Kong, in 2008. His research interests include bioinformatics, machine learning, pattern recognition, intelligent computing and data mining. He has published more than 70 technical articles in referred journals and conference proceedings in the areas of bioinformatics, artificial intelligence, pattern recognition and multimedia. 